\title{
Hölder continuity of singular parabolic equations with variable nonlinearity
}

\author{
Hamid EL BAHJA
}

\begin{abstract}
In this paper we obtain the local Hölder regularity of the weak solutions for singular parabolic equations with variable exponents. The proof is based on DiBenedetto's technique called intrinsic scaling; by choosing an appropriate geometry one can deduce energy and logarithmic estimates from which one can implement an iterative method to obtain the regularity result.
\end{abstract}

\section{Introduction}

Consider the following parabolic equations

$$
u_{t}-\operatorname{div} A(x, t, u, \nabla u)=B(x, t, u, \nabla u) \text { in } \Omega_{T},
$$

where $\Omega_{T} \equiv \Omega \times(0, T], \Omega$ is a bounded simple-connected domain in $\mathbb{R}^{N}$ and $0<T<+\infty$. The functions $A: \Omega_{T} \times \mathbb{R}^{N+1} \longrightarrow \mathbb{R}^{N}$ and $B: \Omega_{T} \times \mathbb{R}^{N+1} \longrightarrow \mathbb{R}$ are assumed to be measurable and satisfying the following structure conditions

$$
\begin{aligned}
& |A(x, t, u, \nabla u)| \leq C_{1}\left(\phi(x, t)+|u|^{p(x, t)-1}+|\nabla u|^{p(x, t)-1}\right), \\
& |B(x, t, u, \nabla u)| \leq C_{2}\left(\phi(x, t)+|u|^{p(x, t)-1}+|\nabla u|^{p(x, t)-1}\right),
\end{aligned}
$$

Key Words: Nonlinear parabolic equations, singular equations, variable nonlinearity, regularity of solutions

2010 Mathematics Subject Classification: Primary 35K55, 35K67; Secondary 35B65.

Received: 10.09.2019

Accepted: 18.12 .2019 


$$
A(x, t, u, \nabla u) . \nabla u \geq C_{3}|\nabla u|^{p(x, t)},
$$

where $\phi(x, t) \in L^{\infty}\left(\Omega_{T}\right)$ and $C_{1}, C_{2}, C_{3}$ are positive constant. Throughout the paper we assume that the exponent $p(x, t)$ is a given measurable function in $\Omega_{T}$ such that

$$
p(x, t) \subset\left[p^{-}, p^{+}\right] \subset(1,2) .
$$

Moreover, we assume that the exponent $p$ satisfies the following log-continuity condition:

$$
\begin{gathered}
|p(x, t)-p(y, \tau)| \leq \frac{c_{0}}{\ln \frac{1}{|t-\tau|+|x-y|}} \text { for any }(x, t),(y, \tau) \in \Omega_{T}, \\
|t-\tau|+|x-y| \leq \frac{1}{2} .
\end{gathered}
$$

The conditions like (1.2)-(1.4) are called $p(x, t)$-growth conditions, which are special cases of nonstandard growth conditions. The parabolic equations with nonstandard growth conditions are the mathematical models of flows of electrorheological or thermorheological fluids, processes of filtration through a porous medium and the processing of digital images [1, 2,9,24]. Further applications of these parabolic equations can be seen in $[4,5,13,20,21]$ or the references therein.

The operator $-\operatorname{div} A(x, t, u, \nabla u)$ is a generalization of the $p(x, t)$-Laplace operator $-\operatorname{div}\left(|\nabla u|^{p(x, t)-2} \nabla u\right)$ and the generalized mean curvature operator $-\operatorname{div}\left(\left(1+|\nabla u|^{2}\right)^{\frac{p(x, t)-2}{2}} \nabla u\right)$ (see [19] for more details). Therefore, the problem (1.1) can be viewed as a generalization of the parabolic $p(x, t)$-Laplace problem

$$
u_{t}-\operatorname{div}\left(|\nabla u|^{p(x, t)-2} \nabla u\right)=B(x, t, u, \nabla u) \text { in } \Omega_{T},
$$

and the generalized parabolic mean curvature problem

$$
u_{t}-\operatorname{div}\left(\left(1+|\nabla u|^{2}\right)^{\frac{p(x, t)-2}{2}} \nabla u\right)=B(x, t, u, \nabla u) \text { in } \Omega_{T} .
$$

For more examples of problems that can be generalized by equation (1.1) see the monograph [4].

Our aim here is to obtain a local regularity result for local weak solutions of (1.1). In order to achieve this goal, and since the equation is singular (the modulus of ellipticity becomes unbounded at points where $|\nabla u|=0$ ), the idea is to study the equation within a geometry that takes this feature into consideration. The building blocks of DiBenedetto's intrinsic scaling method is to show that the continuity of the solution at a point follows from measuring its oscillation in a sequence of nested and shrinking cylinders, with vertex at that point, and showing that the oscillation converge to zero as the cylinders 
shrink to the point. To fully understand the technical procedure, based on the study of an alternative argument which makes use of energy and logarithmic estimates, one has not only to be familiar with DiBenedetto's technique (see $[10,11,22]$ but also to overcome the difficulty of having an $(x, t)$-dependence on the exponent $p$.

The development of the regularity theory for degenerate and/or singular parabolic pdes is one example of the contemporary analysis of nonlinear pdes. One has to go back to the final fifties to encounter the now standard procedure that allows one to get a regularity result for the solutions of nonlinear pdes: regularity for elliptic pdes was established by De Giorgi [12]; while Moser [14, 15, 16, 17], Nash [18] and DiBenedetto [10] dealt with parabolic pdes. Thorough analysis has been carried out for the case of the parabolic isotropic and anisotropic $p(x, t)$ - laplacian, in which Hölder continuity and other properties of solutions are established in $[3,7,8]$.

\section{2 preliminary and main results}

\subsection{Mollification in time}

Since weak solutions of parabolic equations, respectively inequalities possess only weak regularity properties with respect to time, it is in principle not possible to use the solution itself as a test-function in the weak formulation of the problem. In order to be nevertheless able to test properly, there are several possibilities to smooth the solution with respect to the time direction. To overcome these faculties, we consider the Friedrichs mollifier as was done in [3]. Indeed, taking the kernel

$$
\rho \geq 0, \quad \rho \in C_{0}^{\infty}\left(\mathbb{R}^{N}\right), \quad \rho(x) \equiv 0 \quad \text { for }|x| \geq 1, \quad \int_{\mathbb{R}^{N}} \rho(x) d x=1
$$

we introduce regularization of $f \in L_{l o c}^{p(x, t)}\left(\Omega_{T}\right)$ by

$$
\begin{gathered}
I^{h} f=f_{h}(x, t)=h^{-1} \int_{t}^{t+h} \int_{|x-y| \leq h} f(y, \tau) \rho_{h}(x-y) d y d \tau, \\
\rho_{h}(x)=h^{-N} \rho\left(h^{-1} x\right),
\end{gathered}
$$

and consider these inside the cylinder $\Omega_{T}$, i.e., in cylinders $\Omega_{T}^{\prime}=\Omega^{\prime} \times\left(T_{1}, T_{2}\right)$, where $\Omega^{\prime} \subset \Omega, 0<T_{1}<T_{2}<T$. The basic property of the mollification (2.1), which can be retrieved from [[3], Lemma 2.1], is summarized in the following:

Lemma 2.1. If the exponent $p$ satisfies the condition (1.5), then $f_{h} \longrightarrow f$ in $L_{\text {loc }}^{p(x, t)}\left(\Omega_{T}\right)$ as $h \longrightarrow 0$, for any $f \in L_{l o c}^{p(x, t)}\left(\Omega_{T}\right)$. 


\subsection{Formulation of the problem}

We will consider here local weak solutions of equation (1.1), the existence of such solutions is guaranteed by $[23,25]$.

Definition 2.2. A local weak solution of (1.1) is a measurable function $u(x, t)$ defined in $\Omega_{T}$ such that

(i) $u \in C\left(0, T ; L^{2}(\Omega)\right) \cap L^{p^{-}}\left(0, T ; W^{1, p(x, t)}(\Omega)\right)$,

(ii) for every subset $K$ of $\Omega$ and for every subinterval $\left[t_{1}, t_{2}\right]$ of $(0, T]$

$$
\begin{gathered}
{\left[\int_{K} u \phi d x\right]_{t_{1}}^{t_{2}}+\int_{t_{1}}^{t_{2}} \int_{K}\left\{-u \phi_{t}+A(x, t, u, \nabla u) \cdot \nabla \phi\right\} d x d t} \\
=\int_{t_{1}}^{t_{2}} \int_{K} B(x, t, u, \nabla u) \phi d x d t
\end{gathered}
$$

for all locally bounded tested functions

$$
\phi \in W_{l o c}^{1,2}\left(0, T ; L^{2}(K)\right) \cap{L_{l o c}^{p^{-}}}^{\left(0, T ; W_{0}^{1, p(x, t)}(K)\right) .}
$$

We can write (ii) in a way that is technically more convenient and involves the discrete time derivative. This can be accomplished by using the Friedrichs mollifier of a function (see [3] for more details). Then, we get the following:

Lemma 2.3. If $u$ is a solution of equation (1.1) in the sense of Definition 2.2, then for every subset $K$ of $\Omega$, and for any $h<t_{1} \leq t_{2}<T-h$, the following relation

$$
\int_{t_{1}}^{t_{2}} \int_{K}\left\{u_{h, t} \varphi+[A(x, t, u, \nabla u)]_{h} \cdot \nabla \varphi-[B(x, t, u, \nabla u)]_{h} \varphi\right\} d x d t=0,
$$

holds for any locally bounded tested function

$$
\varphi \in W_{l o c}^{1,2}\left(0, T ; L^{2}(K)\right) \cap L_{l o c}^{p^{-}}\left(0, T ; W_{0}^{1, p(x, t)}(K)\right) .
$$

Proof. We introduce the following regularization operator:

$$
I^{-h} f=f_{-h}(x, t)=h^{-1} \int_{t-h}^{t} \int_{|x-y| \leq h} f(y, \tau) \rho_{h}(x-y) d y d \tau .
$$

Consider equation (2.2) with

$$
\phi=I^{-h}(\varphi \chi), \quad \varphi \in W_{l o c}^{1,2}\left(0, T ; L^{2}(K)\right) \cap L_{l o c}^{p^{-}}\left(0, T ; W_{0}^{1, p(x, t)}(K)\right) .
$$


Since

$$
-\int_{t_{1}}^{t_{2}} \int_{K} u \frac{\partial I^{-h}(\varphi \chi)}{\partial t} d x d t=\int_{t_{1}}^{t_{2}} \int_{K} u_{h, t} \varphi \chi d x d t
$$

it follows that

$$
\int_{t_{1}}^{t_{2}} \int_{K}\left[u_{h, t} \varphi \chi+[A(x, t, u, \nabla u)]_{h} \cdot \nabla(\varphi \chi)-[B(x, t, u, \nabla u)]_{h} \varphi \chi\right] d x d t=0 .
$$

Passing here from $\chi \in C_{0}^{\infty}\left(t_{1}, t_{2}\right)$ to characteristic function of the segment $\left[t_{1}, t_{2}\right]$, we obtain the desired relation $(2.3)$.

The proof of the oscillation lemma, which implies the Hölder continuity of solutions $\mathrm{u}(\mathrm{x}, \mathrm{t})$ of equation (1.1), is based on integral estimates for truncated functions of the form

$$
(u-k)_{+}=\max (u-k, 0),(u-k)_{-}=\max (-(u-k), 0), k \in \mathbb{R} .
$$

One of the required inequalities is given in the following lemma.

Lemma 2.4. If $u$ is a solution of equation (1.1) in the sense of Definition 2.2 and $v=(u-k)_{ \pm}$, then for any $h<t_{1} \leq t_{2}<T-h$, the following relation

$$
\int_{t_{1}}^{t_{2}} \int_{\Omega}\left\{v_{h, t} \varphi+[A(x, t, v, \nabla v)]_{h} \cdot \nabla \varphi-[B(x, t, v, \nabla v)]_{h} \varphi\right\} d x d t \leq 0,
$$

holds for any nonnegative tested function

$$
\varphi \in W_{l o c}^{1,2}\left(0, T ; L^{2}(K)\right) \cap{L_{l o c}^{p^{-}}}^{\left(0, T ; W_{0}^{1, p(x, t)}(K)\right) .}
$$

Proof. Taking

$$
\varphi=\frac{\left(u_{h}-k\right)_{ \pm} \phi}{\left(u_{h}-k\right)_{ \pm}+\varepsilon}, \quad \phi \geq 0
$$

where

$$
\frac{\partial \phi}{\partial t} \in L^{2}\left(\Omega_{T}\right) \text {, and } \phi=0 \text { in a neighborhood of } \partial \Omega_{T},
$$

as a test function in (2.2). Integrating by parts in the first term, using (2.1), and passing to the limit $h \longrightarrow 0$ (this is possible due to the interpolation Lemma 2.1) and for $\varepsilon \longrightarrow 0$, we obtain

$$
\begin{aligned}
\int_{t_{1}}^{t_{2}} \int_{\Omega}\left\{-v \frac{\partial \phi}{\partial t}\right. & +A(x, t, v, \nabla v) \cdot \nabla \phi-B(x, t, v, \nabla v) \nabla \phi\} d x d t \\
& \leq-\liminf _{\varepsilon \searrow 0} C \varepsilon \int_{t_{1}}^{t_{2}} \int_{\Omega}|\nabla v|^{p(x)}(v+\varepsilon)^{-2} \phi d x d t .
\end{aligned}
$$


Since the limit is nonnegative and $t_{1}, t_{2}$ are arbitrary, it follows that

$$
\int_{t_{1}}^{t_{2}} \int_{\Omega}\left\{-v \frac{\partial \phi}{\partial t}+A(x, t, v, \nabla v) . \nabla \phi-B(x, t, v, \nabla v) \nabla \phi\right\} d x d t \leq 0 .
$$

Now, repeating the reasoning in the proof of Lemma 2.1, we come to the desired inequality (2.5).

\subsection{Regularity result}

In order to obtain the interior continuity of the solutions by means of intrinsic scaling, we need to consider a geometry that accommodates the singularity of the parabolic equation (1.1). For this purpose, fix a point $\left(x_{0}, t_{0}\right) \in \Omega_{T}$, assume $\left(x_{0}, t_{0}\right)=(0,0)$. Consider a cylinder

$$
Q\left(R^{p^{+}}, R^{\frac{p^{+}}{2}}\right)=K_{R^{\frac{p^{+}}{2}}} \times\left(-R^{p^{+}}, 0\right):=\left\{x: \max _{1 \leq i \leq N}\left|x_{i}\right|<R^{\frac{p^{+}}{2}}\right\} \times\left(-R^{p^{+}}, 0\right)
$$

where $R>0$ is taken such that the inclusion holds. Now, let

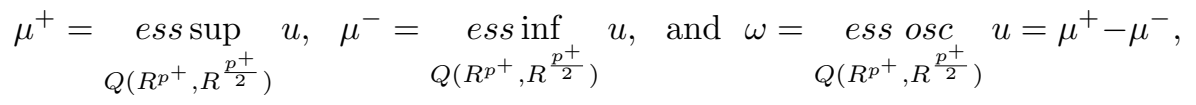

and construct the cylinder

$$
Q\left(R^{p^{+}}, c_{0} R\right) \text { with } c_{0}=\left(\frac{\omega}{2^{\lambda}}\right)^{\frac{p^{-}-2}{p^{+}}}
$$

where $\lambda$ is to determined only in terms of the data.

In order to study our problem inside $Q\left(R^{p^{+}}, R^{\frac{p^{+}}{2}}\right)$, we start the iteration by assuming that

$$
\left(\frac{\omega}{2^{\lambda}}\right)^{\frac{p^{-}-2}{p^{+}}} \leq R^{\frac{p^{+}-2}{2}}
$$

Then, we obtain that

$$
Q\left(R^{p^{+}}, c_{0} R\right) \subset Q\left(R^{p^{+}}, R^{\frac{p^{+}}{2}}\right), \text { and } \underset{Q\left(R^{p^{+}}, c_{0} R\right)}{\operatorname{ess} \text { osc }} u \leq \omega .
$$

Remark 2.5. If (2.6) does not hold, then the essential oscillation $\omega$ goes to zero when the radius $R$ goes to zero, and then there is nothing to prove.

In order to begin our approach, Inside $Q\left(R^{p^{+}}, c_{0} R\right)$ consider subcylinders of smaller size as follows:

$$
(\bar{x}, 0)+Q\left(R^{p^{+}}, d_{0} R\right), \quad d_{0}=\left(\frac{\omega}{2}\right)^{\frac{p^{-}-2}{p^{+}}} .
$$


These cylinders are contained in $Q\left(R^{p^{+}}, c_{0} R\right)$ if $\bar{x}$ ranges over the cube $K_{R(\omega)}$ where

$$
R(\omega)=\left\{\left(2^{\lambda-1}\right)^{\frac{p^{-}-2}{p^{+}}}-1\right\}\left(\frac{\omega}{2}\right)^{\frac{p^{-}-2}{p^{+}}} R=L_{0} d_{0} R,
$$

for $L_{0}=\left(2^{\lambda-1}\right)^{\frac{p^{-}-2}{p^{+}}}-1$.

Now, given $\nu_{0} \in(0,1)$, to be determined in terms of the data, either

$$
\begin{aligned}
& \mid\{(x, t) \in(\bar{x}, 0)\left.+Q\left(R^{p^{+}}, d_{0} R\right): u(x, t)<\mu^{-}+\frac{\omega}{2}\right\} \mid \\
& \leq \nu_{0}\left|Q\left(R^{p^{+}}, d_{0} R\right)\right|
\end{aligned}
$$

or

$$
\begin{aligned}
\mid\{(x, t) \in(\bar{x}, 0) & \left.+Q\left(R^{p^{+}}, d_{0} R\right): u(x, t)<\mu^{-}+\frac{\omega}{2}\right\} \mid \\
& >\nu_{0}\left|Q\left(R^{p^{+}}, d_{0} R\right)\right| .
\end{aligned}
$$

In both cases, we will conclude that the essential oscillation of $u$ within smaller cylinder, centered at the origin, decreases in a way that can be quantitatively measured.

The analysis of this alternative leads to the main result of this work.

Proposition 2.6. There exist constants $\eta \in(0,1)$ and $C, \lambda>1$, that can be determined only on terms of the data, satisfying the following. For n=0,1,2.. construct the sequences

$$
R_{n}=C^{-n} R, \omega_{n}=\eta^{n} \omega
$$

and the cylinders

$$
Q_{n}=Q\left(R_{n}^{p^{+}}, c_{n} R_{n}\right) \text { with } c_{n}=\left(\frac{\omega_{n}}{2^{\lambda}}\right)^{\frac{p^{-}-2}{p^{+}}} .
$$

Then, for all $n=0,1,2$. ,

$$
Q_{n+1} \subset Q_{n} \text { and ess osc } u \leq \omega_{n} .
$$

As an immediate consequence, we obtain the following.

Theorem 2.7. Under assumptions (1.2)-(1.5), any locally bounded weak solution of (1.1) is locally Hölder continuous.

Remark 2.8. By using the result in Proposition 2.6, the proof of Theorem 2.7 follows from a slight modification of the argument in Theorem 1.1 in [3]. 


\section{$3 \quad$ Local energy and logarithmic estimates}

Let $\tau$ and $\rho$ be so small that $Q(\tau, \rho) \subset \Omega_{T}$. Let $\xi$ denote a piecewise smooth cutoff function in $Q(\tau, \rho)$ such that

$$
\xi \in[0,1], \quad|\nabla \xi|<\infty \text { and } \xi(x, t)=0 \text { for } x \text { outside } K_{\rho} .
$$

Proposition 3.1. Let $u$ be a local weak solution of (1.1) in $\Omega_{T}$. There exist positive constants $C$ and $C^{\prime}$ such that, for every cylinder $Q(\tau, \rho) \subset \Omega_{T}$ and for every $k \in \mathbb{R}$

$$
\begin{aligned}
& \sup _{-\tau<t<0} \int_{K_{\rho}}(u-k)_{ \pm}^{2} \xi^{p^{+}}(x, t) d x+C \int_{-\tau}^{t} \int_{K_{\rho}}\left|\nabla(u-k)_{ \pm}\right|^{p^{-}} \xi^{p^{+}} d x d t \\
& \leq \int_{K_{\rho}}(u-k)_{ \pm}^{2} \xi^{p^{+}}(x,-\tau) d x+C^{\prime}\left[\int_{-\tau}^{t} \int_{K_{\rho}}(u-k)_{ \pm}^{2} \xi^{p^{+}-1} \xi_{t} d x d t\right. \\
& +\int_{-\tau}^{t} \int_{K_{\rho}}(u-k)_{ \pm}^{p^{+}}\left(|\nabla \xi|^{p^{+}}+\xi^{p^{+}}\right) d x d t \\
& \left.+\int_{-\tau}^{t} \int_{K_{\rho}} \chi\left((u-k)_{ \pm}>0\right) d x d t\right] .
\end{aligned}
$$

Proof. See Proposition 3.1 in [7]

Now, introduce the logarithmic function

$$
\psi^{ \pm}(u)=\psi\left(H_{k}^{ \pm},(u-k)_{ \pm}, c\right)=\left(\ln \left(\frac{H_{k}^{ \pm}}{H_{k}^{ \pm}-(u-k)_{ \pm}+c}\right)\right)_{+},
$$

where $H_{k}^{ \pm}=e s s \sup _{Q(\tau, \rho)}\left|(u-k)_{ \pm}\right|$and $0<c<H_{k}^{ \pm}$. In the cylinder $Q(\tau, \rho)$, we take a cutoff function satisfying $\xi \in[0,1],|\nabla \xi|<\infty$ and $\xi$ is independent of $t \in(-\tau, 0)$.

Proposition 3.2. Let $u$ be local weak solution of (1.1) in $\Omega_{T}$. There exists a positive constant $C$ such that for every cylinder $Q(\tau, \rho) \in \Omega_{T}$ and for every 
level $k \in \mathbb{R}$

$$
\begin{aligned}
& \text { esssup } \int_{-\tau<t<0}\left[\psi_{K_{\rho}}^{ \pm}(u)\right]^{2} \xi^{p^{+}} d x \\
& \leq \int_{K_{\rho} \times\{-\tau\}}\left[\psi^{ \pm}(u)\right]^{2} \xi^{p^{+}} d x+C\left(\int_{-\tau}^{0} \int_{K_{\rho}} \psi^{ \pm}(u)\left[\left(\psi^{ \pm}\right)^{\prime}(u)\right]^{2} \xi^{p^{+}} d x d t\right. \\
& +\int_{-\tau}^{0} \int_{K_{\rho}} \psi^{ \pm}(u)\left[\left(\psi^{ \pm}\right)^{\prime}(u)\right]^{2-p^{-}}\left(|\nabla u|^{p^{+}}+1+\xi^{p^{+}}\right) d x d t \\
& +\int_{-\tau}^{0} \int_{K_{\rho}} \psi^{ \pm}(u)\left(|\nabla u|^{p^{+}}+1+\xi^{p^{+}}\right) d x d t \\
& \left.+\int_{-\tau}^{0} \int_{K_{\rho}}|u|^{p^{+}} \psi^{ \pm}(u)\left[\left(\psi^{ \pm}\right)^{\prime}(u)\right]^{2} \xi^{p^{+}} d x d t\right) .
\end{aligned}
$$

Proof. See Proposition 3.2 in [7]

\section{Rescaled iteration}

The following rescaled iteration technique applies to any subcylinder of $\Omega_{T}$ and it is crucial in both alternatives (2.9) and (2.10). Let $m>0$ be given by $m=m_{1}+m_{2}$, where $m_{1} \geq 1$, and $m_{2} \geq 0$ and consider the cube

$$
K_{d_{1} R}=\left\{x \in \mathbb{R}^{\mathbb{N}}, \max _{1 \leq i \leq N}\left|x_{i}\right|<d_{1} R\right\}, \quad d_{1}=\left(\frac{\omega}{2^{m_{1}}}\right)^{\frac{p^{-}-2}{p^{+}}},
$$

and the box

$$
Q_{R}\left(m_{1}, m_{2}\right)=K_{d_{1} R} \times\left(-2^{m_{2}\left(p^{-}-2\right)} R^{p^{+}}, 0\right) .
$$

Fix $(\bar{x}, \bar{t}) \in \Omega_{T}$, and let $R>0$ be so small that

$$
(\bar{x}, \bar{t})+Q_{R}\left(m_{1}, m_{2}\right) \subset \Omega_{T} .
$$

Lemma 4.1. There exists a number $\nu_{0}$ that can be determined a priori only in terms of the data, such that:

(1) If $u$ is a super-solution of (1.1) in $(\bar{x}, \bar{t})+Q_{R}\left(m_{1}, m_{2}\right)$ satisfying

$$
\underset{(\bar{x}, \bar{t})+Q_{R}\left(m_{1}, m_{2}\right)}{\operatorname{ess} \operatorname{osc}} u \leq \omega
$$

and

$$
\left|\left\{(x, t) \in(\bar{x}, \bar{t})+Q_{R}\left(m_{1}, m_{2}\right): u(x, t)<\mu^{-}+\frac{\omega}{2^{m}}\right\}\right| \leq \nu_{0}\left|Q_{R}\left(m_{1}, m_{2}\right)\right|,
$$


then

$$
u(x, t) \geq \mu^{-}+\frac{\omega}{2^{m+1}}, \quad \forall(x, t) \in(\bar{x}, \bar{t})+Q_{\frac{R}{2}}\left(m_{1}, m_{2}\right) .
$$

(2) If $u$ is a sub-solution of (1.1) in $(\bar{x}, \bar{t})+Q_{R}\left(m_{1}, m_{2}\right)$ satisfying

$$
\underset{(\bar{x}, \bar{t})+Q_{R}\left(m_{1}, m_{2}\right)}{\operatorname{ess} \operatorname{osc}} u \leq \omega
$$

and

$\left|\left\{(x, t) \in(\bar{x}, \bar{t})+Q_{R}\left(m_{1}, m_{2}\right): u(x, t)>\mu^{+}-\frac{\omega}{2^{m}}\right\}\right| \leq \nu_{0}\left|Q_{R}\left(m_{1}, m_{2}\right)\right|$,

then

$$
u(x, t) \leq \mu^{+}-\frac{\omega}{2^{m+1}}, \quad \forall(x, t) \in(\bar{x}, \bar{t})+Q_{\frac{R}{2}}\left(m_{1}, m_{2}\right) .
$$

Proof. We only prove the statement regarding super-solutions (for sub-solutions the proof is similar). Assume $(\bar{x}, \bar{t})=(0,0)$ and construct the decreasing sequences of numbers

$$
R_{n}=\frac{R}{2}+\frac{R}{2^{n+1}}, k_{n}=\mu^{-}+\frac{\omega}{2^{m+1}}+\frac{\omega}{2^{m+n+1}}, n=0,1,2, . .
$$

and the families of nested cubes and cylinders

$$
\begin{gathered}
K_{n}=K_{d_{1} R_{n}}, d_{1}=\left(\frac{\omega}{2^{m_{1}}}\right)^{\frac{p^{-}-2}{p^{+}}}, \\
Q_{n}=Q_{R_{n}}\left(m_{1}, m_{2}\right)=K_{n} \times\left(-2^{\left(p^{-}-2\right) m_{2}} R_{n}^{p^{+}}, 0\right) .
\end{gathered}
$$

Let $0<\xi_{n}(x, t) \leq 1$ be piecewise smooth functions in $Q_{n}$ such that

$$
\left\{\begin{array}{l}
\xi_{n}=1 \text { in } Q_{n+1}, \xi_{n}=0 \text { on } \partial Q_{n}, \\
\left|\nabla \xi_{n}\right| \leq \frac{2^{n+2}}{R}\left(\frac{\omega}{2^{m_{1}}}\right)^{\frac{2-p^{-}}{p^{+}}} \text {, and } 0 \leq \xi_{n, t} \leq 2^{\left(2-p^{-}\right) m_{2}} \times \frac{2^{p^{+}(n+2)}}{R^{p^{+}}} .
\end{array}\right.
$$


Now, by using the energy inequality (3.1) for the function $\left(u-k_{n}\right)_{-}$we get

$$
\begin{aligned}
\sup _{-2^{\left(p^{-}-2\right) m_{2} R_{n}^{p^{+}}<t<0}} & \int_{K_{n}}\left(u-k_{n}\right)_{-}^{2} \xi_{n}^{p^{+}}(x, t) d x \\
& +\iint_{Q_{n}}\left|\nabla\left(u-k_{n}\right)_{-}\right|^{p^{-}} \xi_{n}^{p^{+}} d x d t \\
& \leq C\left[\frac{2^{\left(2-p^{-}\right) m_{2} 2^{p^{+}(n+2)}}}{R^{p^{+}}} \iint_{Q_{n}}\left(u-k_{n}\right)_{-}^{2} d x d t\right. \\
& +\frac{2^{(n+2) p^{+}}}{R^{p^{+}}}\left(\frac{\omega}{2^{m_{1}}}\right)^{2-p^{-}} \iint_{Q_{n}}\left(u-k_{n}\right)_{-}^{p^{+}} d x d t \\
& \left.+\iint_{Q_{n}} \chi_{\left(\left(u-k_{n}\right)_{-}>0\right)} d x d t\right] .
\end{aligned}
$$

Since the solutions are assumed to be bounded we can always normalize and assume, w.l.g., that

$$
\omega \leq 1
$$

then

Also, by using the fact that

$$
\frac{\omega}{2^{m_{1}}}<1 \Longrightarrow\left(\frac{\omega}{2^{m_{1}}}\right)^{2-p^{-}} \leq\left(\frac{\omega}{2^{m_{1}}}\right)^{2-p^{+}} .
$$

$$
\begin{gathered}
\left(u-k_{n}\right)_{-} \leq \sup _{Q_{n}}\left(u-k_{n}\right)_{-} \leq \frac{\omega}{2^{m+1}}+\frac{\omega}{2^{m+1+n}} \leq \frac{\omega}{2^{m}}, \\
\left(\frac{\omega}{2^{m_{1}}}\right)^{2-p^{+}}\left(\frac{\omega}{2^{m}}\right)^{p^{+}}=\left(\frac{\omega}{2^{m}}\right)^{2} 2^{\left(2-p^{+}\right) m_{2}},
\end{gathered}
$$

and by means of assumption (2.6) and Remark 2.5, the terms on the right hand side of (4.1) are estimated above by

$$
C 2^{\left(2-p^{-}\right) m_{2}} \frac{2^{n p^{+}}}{R^{p^{+}}}\left(\frac{\omega}{2^{m}}\right)^{2} \iint_{Q_{n}} \chi_{\left(\left(u-k_{n}\right)_{-}>0\right)} d x d t .
$$

Setting

$$
\bar{k}_{n}=\frac{k_{n}+k_{n+1}}{2},
$$

then for all $t \in\left(-2^{\left(2-p^{-}\right) m_{2}} R_{n}^{p^{+}}, 0\right)$, we have

$$
\begin{array}{r}
\int_{K_{n}}\left(u-k_{n}\right)_{-}^{2} \xi_{n}^{p^{+}}(x, t) d x \geq \int_{K_{n}}\left(k_{n}-\bar{k}_{n}\right)^{2-p^{-}}\left(u-k_{n}\right)_{-}^{p^{-}} \xi_{n}^{p^{+}}(x, t) d x \\
=\left(\frac{\omega}{2^{m}}\right)^{2-p^{-}} 2^{(n+3)\left(p^{-}-2\right)} \int_{K_{n}}\left(u-\bar{k}_{n}\right)_{-}^{p^{-}} \xi_{n}^{p^{+}}(x, t) d x
\end{array}
$$


and since $k_{n}>\bar{k}_{n}$, we have

$$
\iint_{Q_{n}}\left|\nabla\left(u-k_{n}\right)_{-}\right|^{p^{-}} \xi_{n}^{p^{+}} d x d t \geq \iint_{Q_{n}}\left|\nabla\left(u-\bar{k}_{n}\right)_{-}\right|^{p^{-}} \xi_{n}^{p^{+}} d x d t .
$$

By combining all the previous estimates and dividing through by

$$
\left(\frac{\omega}{2^{m}}\right)^{2-p^{-}} 2^{(n+3)\left(p^{-}-2\right)},
$$

we obtain

$$
\begin{aligned}
& \sup _{-2^{\left(p^{-}-2\right) m_{2}} R_{n}^{p^{+}}<t<0} \int_{K_{n}}\left(u-\bar{k}_{n}\right)_{-}^{p^{-}} \xi_{n}^{p^{+}} d x \\
& +\left(\frac{\omega}{2^{m}}\right)^{p^{-}-2} 2^{(n+3)\left(2-p^{-}\right)} \iint_{Q_{n}}\left|\nabla\left(u-\bar{k}_{n}\right)_{-}\right|^{p^{-}} \xi_{n}^{p^{+}} d x d t \\
& \leq C \frac{2^{2 n}}{R^{p^{+}}} 2^{\left(2-p^{-}\right) m_{2}}\left(\frac{\omega}{2^{m}}\right)^{p^{-}} \iint_{Q_{n}} \chi_{\left(\left(u-k_{n}\right)_{-}>0\right)} d x d t .
\end{aligned}
$$

Next consider the change of variables

$$
y=d_{1}^{-1} x, z=2^{\left(2-p^{-}\right) m_{2}} t
$$

which maps the cylinders $Q_{n}$ into the cylinders $Q_{n}^{\prime}=K_{R_{n}} \times\left(-R_{n}^{p^{+}}, 0\right)$, and define new functions

$$
\hat{u}(y, z)=u\left(d_{1} y, 2^{\left(p^{-}-2\right) m_{2}} z\right), \hat{\xi}_{n}=\xi_{n}\left(d_{1} y, 2^{\left(p^{-}-2\right) m_{2}} z\right),
$$

and the sets

$$
A_{n}(z)=\left\{y \in K_{R_{n}}: \hat{u}(y, z)<K_{n}\right\}, \text { with }\left|A_{n}\right|=\int_{-R_{n}^{p^{+}}}^{0}\left|A_{n}(z)\right| d z .
$$

Since $1<p^{-} \leq p(x) \leq p^{+}<2$ and $\omega<1$, the coefficient

$$
\left(\frac{\omega}{2^{m}}\right)^{p^{-}-2} 2^{(n+3)\left(2-p^{-}\right)+m_{2}\left(p^{-}-2\right)}=\omega^{p^{-}-2} 2^{\left(2-p^{-}\right)\left(m_{1}+n+3\right)}>1,
$$

then (4.3) becomes

$$
\begin{gathered}
\sup _{-R_{n}^{p^{+}}<t<0} \int_{K_{R_{n}}}\left(\hat{u}-\bar{k}_{n}\right)_{-}^{p^{-}} \hat{\xi}_{n}^{p^{+}} d z+\iint_{Q_{n}^{\prime}}\left|\nabla\left(\hat{u}-\bar{k}_{n}\right)_{-}\right|^{p^{-}} \hat{\xi}_{n}^{p^{+}} d y d z \\
\leq C \frac{2^{2 n}}{R^{p^{+}}}\left(\frac{\omega}{2^{m}}\right)^{p^{-}} \iint_{Q_{n}^{\prime}} \chi_{\left(\left(\hat{u}-k_{n}\right)_{-}>0\right)} d y d z .
\end{gathered}
$$


By Hölder's inequality, Proposition 3.1 of chapter I in [10], and (4.4), we obtain

$$
\begin{aligned}
& \int_{-R_{n}^{p^{+}}}^{0} \int_{K_{R_{n}}}\left(\hat{u}-\bar{k}_{n}\right)_{-}^{p^{-}} \hat{\xi}_{n}^{p^{+}} d y d z \\
& \leq\left\{\int_{-R_{n}^{p+}}^{0} \int_{K_{R_{n}}}\left[\left(\hat{u}-\overline{k_{n}}\right)_{-}{\hat{\xi_{n}}}^{\frac{p^{+}}{p^{-}}}\right]^{\frac{p^{-}\left(N+p^{-}\right)}{N}} d y d z\right\}^{\frac{N}{N+p^{-}}} \\
& \times\left\{\int_{-R_{n}^{p^{+}}}^{0} \int_{K_{R_{n}}} \chi_{\left(\left(\hat{u}-k_{n}\right)_{-}>0\right)}^{\frac{N+p^{-}}{p^{-}}} d y d z\right\}^{\frac{p^{-}}{N+p^{-}}} \\
& =\left\{\int_{-R_{n}^{p^{+}}}^{0} \int_{K_{R_{n}}}\left[\left(\hat{u}-\overline{k_{n}}\right)_{-}{\hat{\xi_{n}}}^{\frac{p^{+}}{p^{-}}}\right]^{\frac{p^{-}\left(N+p^{-}\right)}{N}} d y d z\right\}^{\frac{N}{N+p^{-}}}\left|A_{n}\right|^{\frac{p^{-}}{N+p^{-}}} \\
& \leq C\left[\sup _{-R_{n}^{p^{+}}<z<0} \int_{K_{R_{n}}}\left(\hat{u}-\overline{k_{n}}\right)_{-}^{p^{-}}{\hat{\xi_{n}}}^{p^{+}} d y\right]^{\frac{p^{-}}{N+p^{-}}} \\
& \times\left[\int_{-R_{n}^{p^{+}}}^{0} \int_{K_{R_{n}}}\left|\nabla\left(\hat{u}-\overline{k_{n}}\right)_{-}\right|^{p^{-}}{\hat{\xi_{n}}}^{p^{+}} d y d z\right. \\
& \left.+\int_{-R_{n}^{p^{+}}}^{0} \int_{K_{R_{n}}}\left(\hat{u}-\overline{k_{n}}\right)_{-}^{p^{-}}\left|\nabla \hat{\xi_{n}}\right|^{p^{-}} d y d x\right]^{\frac{N}{N+p^{-}}}\left|A_{n}\right|^{\frac{p^{-}}{N+p^{-}}} \\
& \leq C \frac{2^{2 n}}{R^{p^{+}}}\left(\frac{\omega}{2^{m}}\right)^{p^{-}}\left|A_{n}\right|^{1+\frac{p^{-}}{N+p^{-}}} .
\end{aligned}
$$

On the other hand

$$
\begin{aligned}
\int_{-R_{n}^{p^{+}}}^{0} \int_{K_{R_{n}}}\left(\hat{u}-\overline{k_{n}}\right)_{-}^{p^{-}} \hat{\xi}_{n}^{p^{+}} d y d z & \geq \int_{-R_{n+1}^{p^{+}}}^{0} \int_{K_{R_{n+1}}}\left(\hat{u}-\overline{k_{n}}\right)_{-}^{p^{-}} d y d z \\
& \geq\left|\overline{k_{n}}-k_{n+1}\right|^{p^{-}}\left|A_{n+1}\right| \\
& =2^{-(n+3) p^{-}}\left(\frac{\omega}{2^{m}}\right)^{p^{-}}\left|A_{n+1}\right| .
\end{aligned}
$$

Combining (4.5) and (4.6), we get that

$$
\left|A_{n+1}\right| \leq C 4^{n p^{+}}\left|A_{n}\right|^{1+\frac{p^{-}}{N+p^{-}}} .
$$

Next, by defining the numbers

$$
Y_{n}=\frac{\left|A_{n}\right|}{\left|Q_{n}^{\prime}\right|},
$$


we obtain the following recursive relation

$$
Y_{n+1} \leq C 4^{n p^{+}} Y_{n}^{1+\frac{p^{-}}{N+p^{-}}}
$$

Therefore, Lemma 4.1 of Chapter I in [10] implies that if

$$
Y_{0} \leq C^{-\frac{N+p^{-}}{p^{-}}} 4^{-p^{+}\left(\frac{p^{-}+N}{p^{-}}\right)^{2}}=\nu_{0}
$$

then

$$
Y_{n} \longrightarrow 0 \text {. }
$$

However, (4.8) is nothing but the assumption (2.9). Hence, the result easily follows from (4.9).

\section{5 continuity of the weak solutions}

In this section we analyze the alternative and prove proposition 2.6. Assume that these exists a cylinder of the type $\left[(\bar{x}, 0)+Q\left(R^{p^{+}}, d_{0} R\right)\right]$ making up the partition of $Q\left(R^{p^{+}}, c_{0} R\right)$ for which $(2.9)$ holds. Then by applying Lemma 4.1 with $m_{1}=1$ and $m_{2}=0$, we conclude that

$$
u(x, t) \geq \mu^{-}+\frac{\omega}{4}, \forall(x, t) \in(\bar{x}, 0)+Q\left(\left(\frac{R}{2}\right)^{p^{+}}, d_{0} \frac{R}{2}\right) .
$$

We view the box $\left[(\bar{x}, 0)+Q\left(\left(\frac{R}{2}\right)^{p^{+}}, d_{0} \frac{R}{2}\right)\right]$ as a block inside $Q\left(R^{p^{+}}, c_{0} R\right)$. Let $R(\omega)$ be the radius introduced in (2.8). The location of $\bar{x}$ within the cube $K_{R(\omega)}$ is only known qualitatively. We will show that the positivity of $(5.1)$ spreads over the full cube $K_{c_{0} R}$, for all times

$$
-\left(\frac{R}{8}\right)^{p^{+}} \leq t \leq 0
$$

In a precise way we will prove the following

Proposition 5.1. Assume that (2.9) holds for some $\bar{x} \in K_{R(\omega)}$. There exists a positive number $s_{1}$ that can be determined a priori in terms of the data, such that

$$
u(x, t) \geq \mu^{-}+\frac{\omega}{2^{s_{1}+1}}, \forall(x, t) \in Q\left(\left(\frac{R}{8}\right)^{p^{+}}, c_{0} R\right)
$$


To prove Proposition 5.1, we regard $\bar{x}$ as the center of a large cube $\bar{x}+K_{8 c_{0} R}$ which we may assume to be contained in $K_{R^{\frac{p^{+}}{2}}}$. Indeed if not we could have

$$
16 c_{0} R>R^{\frac{p^{+}}{2}} \Longrightarrow \omega \leq 16^{\frac{p^{+}}{2-p^{-}}} 2^{\lambda} R^{\frac{p^{+}}{2}}
$$

then there will be nothing to prove. We work within the box

$$
(\bar{x}, 0)+Q\left(\left(\frac{R}{2}\right)^{p^{+}}, 8 c_{0} R\right)
$$

and show that the conclusion of Proposition 5.1 holds within the cylinder

$$
(\bar{x}, 0)+Q\left(\left(\frac{R}{8}\right)^{p^{+}}, 2 c_{0} R\right) .
$$

This contains $Q\left(\left(\frac{R}{8}\right)^{p^{+}}, c_{0} R\right)$, regardless of the location of $\bar{x}$ in the cube $K_{R(\omega)}$.

We begin by considering an auxiliary function

$$
v=\left(u-\mu^{-}\right) \frac{2}{\omega},
$$

and introducing the following change of variables

$$
x \longmapsto \frac{x-\bar{x}}{2 c_{0} R}, \quad t \longmapsto\left(\frac{8}{R}\right)^{p^{+}} t .
$$

Thus, the cylinder $(\bar{x}, 0)+Q\left(\left(\frac{R}{2}\right)^{p^{+}}, 8 c_{0} R\right)$ is mapped into $Q_{4}=K_{4} \times$ $\left(-4^{p^{+}}, 0\right)$. Denoting again with $x$ and $t$ the new variables, the function $v$ satisfies the PDE

$$
\frac{\partial v}{\partial t}-\operatorname{div} \tilde{A}(x, t, v, \nabla v)=\tilde{B}(x, t, v, \nabla v), \text { in } D^{\prime}\left(Q_{4}\right),
$$

with

$$
\tilde{A}(x, t, v, \nabla v)=\left(\frac{8}{R}\right)^{-p^{+}}\left(\frac{\omega}{2}\right)^{-1} \frac{1}{2 c_{0} R} A(x, t, u, \nabla u)
$$

and

$$
\tilde{B}(x, t, v, \nabla v)=\left(\frac{8}{R}\right)^{-p^{+}}\left(\frac{\omega}{2}\right)^{-1} B(x, t, u, \nabla u) .
$$


By using (1.2)-(1.4) and assumptions (2.6) and (4.2), the applications $\tilde{A}$ : $Q_{4} \times \mathbb{R}^{N+1} \longrightarrow \mathbb{R}^{N}$ and $\tilde{B}: Q_{4} \times \mathbb{R}^{N+1} \longrightarrow \mathbb{R}^{N}$ satisfies the following structure conditions

$$
\begin{aligned}
& |\tilde{A}(x, t, v, \nabla v)| \leq C\left(\tilde{\phi}(x, t)+|v|^{p(x, t)-1}+|\nabla v|^{p(x, t)-1}\right), \\
& |\tilde{B}(x, t, v, \nabla v)| \leq C\left(\tilde{\phi}(x, t)+|v|^{p(x, t)-1}+|\nabla v|^{p(x, t)-1}\right), \\
& \tilde{A}(x, t, v, \nabla v) . \nabla v \geq C|\nabla v|^{p(x, t)}
\end{aligned}
$$

where $\tilde{\phi}(x, t) \in L^{\infty}\left(\Omega_{T}\right)$ and $C$ is a positive constant depend on $p^{+}, p^{-}, \lambda$ and $R$. Therefore, (5.1) becomes

$$
v(x, t) \geq \frac{1}{2} \text { a.e. }(x, t) \in Q\left(h_{0}\right)=\left\{x:|x|<h_{0}\right\} \times\left(-4^{p^{+}}, 0\right)
$$

where

$$
h_{0}=\frac{d_{0}}{4 c_{0}}=\frac{1}{4}\left(\frac{2}{2^{\lambda}}\right)^{\frac{2-p^{-}}{p^{+}}}=2^{\frac{(\lambda-1)\left(p^{-}-2\right)}{p^{+}}-2}<1 .
$$

We regard $Q\left(h_{0}\right)$ as a thin cylinder sitting at the center of $Q_{4}$. We will prove that the relative largeness of $v$ in $Q\left(h_{0}\right)$ spreads sidewise over $Q_{2}$.

Proposition 5.1 will be a consequence of the following lemma.

Lemma 5.2. For every $\nu \in(0,1)$ there exists a positive number $\delta^{*} \in(0,1)$, that can be determined a priori only in terms of $\nu, N, p^{+}, p^{-}$and the data, such that

$$
\left|\left\{x \in K_{2}: v(x, t) \leq \delta^{*}\right\}\right| \leq \nu\left|K_{2}\right|,
$$

for all time levels $t \in\left[-2 p^{+}, 0\right]$.

Proof. The weak formulation of (5.5) is

$$
\int_{K_{4}} v_{t} \varphi d x+\int_{K_{4}} \tilde{A}(x, t, v, \nabla v) \cdot \nabla \varphi d x=\int_{K_{4}} \tilde{B}(x, t, v, \nabla v) \varphi d x
$$

for all $-4^{p^{+}}<t<0$ and all testing functions

$$
\varphi \in W^{1,2}\left(-4^{p^{+}}, 0 ; L^{2}\left(K_{4}\right)\right) \cap L^{p(x, t)}\left(-4^{p^{+}}, 0 ; W_{0}^{1, p(x, t)}\left(K_{4}\right)\right) .
$$

Taking into account the structure of the function $v$ and using Lemma 2.4, we can claim that the truncated function $(k-v)_{+}$for all positive testing function $\varphi$, satisfies the following

$$
\begin{aligned}
\int_{K_{4}} \frac{\partial}{\partial \tau}(k-v)_{+} \varphi(x, t) d x & +\int_{K_{4}} \tilde{A}\left(x, t, v, \nabla\left((k-v)_{+}\right)\right) \cdot \nabla \varphi d x \\
& \leq \int_{K_{4}} \tilde{B}\left(x, t, v, \nabla\left((k-v)_{+}\right)\right) \varphi d x
\end{aligned}
$$


In this formulation we take the testing function

$$
\varphi=\frac{\xi^{p^{+}}}{\left[k-(k-v)_{+}+\delta k+R\right]^{p^{-}-1}},
$$

where $\xi=\xi_{1}(x) \xi_{2}(t)$ is a piecewise smooth cutoff function in $Q_{4}$ satisfying

$$
\left\{\begin{array}{l}
0 \leq \xi \leq 1 \text { in } Q_{4}, \text { and } \xi=1 \text { in } Q_{2}, \\
\xi=0 \text { on the parabolic boundary of } Q_{4}, \\
\left|\nabla \xi_{1}\right| \leq 1,0 \leq \xi_{2, t} \leq 1, \\
\text { the set }\left\{x \in K_{4} \mid \xi_{1}(x)>k\right\} \text { are convex } \forall k \in(0,1) .
\end{array}\right.
$$

Set also

$$
\left\{\begin{array}{l}
\phi_{k}(v)=\int_{0}^{(k-v)_{+}} \frac{d s}{[(1+\delta) k-s+R]^{p^{-}-1}} \\
\psi_{k}(v)=\ln \left[\frac{k(1+\delta)+R}{(1+\delta) k-(k-v)_{+}+R}\right] .
\end{array}\right.
$$

Here $k \in\left(0, \frac{1}{8}\right), \delta \in\left(0, \frac{1}{8}\right)$ and $R \leq k \delta^{\frac{p^{-}}{p^{-}-1}}$. Then, we obtain

$$
\begin{aligned}
& \int_{K_{4}} \frac{\partial}{\partial t}(k-v)_{+} \frac{\xi^{p^{+}}}{\left[k(1+\delta)-(k-v)_{+}+R\right]^{p^{-}-1}} d x \\
& \quad+\int_{K_{4}} \tilde{A}\left(x, t, v, \nabla(k-v)_{+}\right) \cdot\left[\frac{\left(p^{-}-1\right) \nabla(k-v)_{+} \xi^{p^{+}}}{\left[k(1+\delta)-(k-v)_{+}+R\right]^{p^{-}}}\right. \\
& \left.\quad+\frac{p^{+} \xi^{p^{+}-1} \nabla \xi}{\left[k(1+\delta)-(k-v)_{+}+R\right]^{p^{-}-1}}\right] d x \\
& \quad \leq \int_{K_{4}} \tilde{B}\left(x, t, v, \nabla(k-v)_{+}\right) \frac{\xi^{p^{+}}}{\left[k(1+\delta)-(k-v)_{+}+R\right]^{p^{-}-1}} d x
\end{aligned}
$$

Since $u$ is bounded, by simple computation we get that

$$
\begin{aligned}
0 \leq C_{1}\left(p^{+}, p^{-}, \delta, k\right) & \leq\left[k(1+\delta)-(k-v)_{+}+R\right] \\
& \leq C_{2}\left(p^{+}, p^{-}, \delta, k\right)<\infty
\end{aligned}
$$

Now, estimating the various terms separately. Then by using (5.8)-(5.10), (5.20) and $\tilde{\phi}(x, t) \in L^{\infty}\left(\Omega_{T}\right)$, we obtain the following

$$
\begin{aligned}
\int_{K_{4}} \frac{\partial}{\partial t} \phi_{k}(v) \xi^{p^{+}} d x= & -\int_{K_{4}} \phi_{k}(v) p^{+} \xi^{p^{+}-1} \xi_{t} d x \\
& +\frac{\partial}{\partial t} \int_{K_{4}} \phi_{k}(v) \xi^{p^{+}} d x
\end{aligned}
$$




$$
\begin{aligned}
& \int_{K_{4}} \frac{\tilde{A}\left(x, t, v, \nabla(k-v)_{+}\right) \nabla(k-v)_{+} \xi^{p^{+}}}{\left[k(1+\delta)-(k-v)_{+}+R\right]^{p^{-}}} d x \\
& \geq C \int_{K_{4}} \frac{\left|\nabla(k-v)_{+}\right|^{p(x, t)} \xi^{p^{+}}}{\left[k(1+\delta)-(k-v)_{+}+R\right]^{p^{-}}} d x \\
& =C \int_{K_{4}} \frac{\left|\nabla(k-v)_{+}\right|^{p(x, t)} \xi^{p^{+}}}{\left[k(1+\delta)-(k-v)_{+}+R\right]^{p(x, t)}} \\
& \quad \times\left[k(1+\delta)-(k-v)_{+}+R\right]^{p(x, t)-p^{-}} d x \\
& \geq C \int_{K_{4}}\left|\nabla \psi_{k}(v)\right|^{p(x, t)} \xi^{p^{+}} d x,
\end{aligned}
$$

also by using Young's inequality, and the fact that $0 \leq \xi \leq 1$ and $\frac{p(x, t)}{p(x, t)-1} \geq$ $\frac{p^{+}}{p^{+}-1}$ imply that $\xi^{\frac{p(x, t)\left(p^{+}-1\right)}{p(x, t)-1}} \leq \xi^{p^{+}}$, we get that

$$
\begin{aligned}
& \int_{K_{4}} \frac{\tilde{A}\left(x, t, v, \nabla(k-v)_{+}\right) \xi^{p^{+}-1} \nabla \xi}{\left[k(1+\delta)-(k-v)_{+}+R\right]^{p^{-}-1}} d x \\
& \leq C \int_{K_{4}} \frac{\left(\tilde{\phi}(x, t)+\left|(k-v)_{+}\right|^{p(x, t)-1}+\left|\nabla(k-v)_{+}\right|^{p(x, t)-1}\right)}{\left[k(1+\delta)-(k-v)_{+}+R\right]^{p^{-}-1}} \\
& \times \xi^{p^{+}-1}|\nabla \xi| d x \\
& \leq C\left(\int_{K_{4}} \frac{\xi^{p^{+}-1}|\nabla \xi|}{\left[k(1+\delta)-(k-v)_{+}+R\right]^{p^{-}-1}} d x\right. \\
& \left.+\int_{K_{4}}\left|\nabla \psi_{k}(v)\right|^{p(x, t)-1} \xi^{p^{+}-1}|\nabla \xi| d x\right) \\
& \leq C\left(\varepsilon \int_{K_{4}}\left|\nabla \psi_{k}(v)\right|^{p(x, t)} \xi^{p^{+}} d x+C(\varepsilon) \int_{K_{4}}|\nabla \xi|^{p(x, t)} d x\right. \\
& \left.+\int_{K_{4}} \frac{\xi^{p^{+}-1}|\nabla \xi|}{\left[k(1+\delta)-(k-v)_{+}+R\right]^{p^{-}-1}} d x\right) .
\end{aligned}
$$


By the same method, the term on the right hand side of (5.15) becomes

$$
\begin{aligned}
& \int_{K_{4}} \tilde{B}\left(x, t, v, \nabla(k-v)_{+}\right) \frac{\xi^{p^{+}}}{\left[k(1+\delta)-(k-v)_{+}+R\right]^{p^{-}-1}} d x \\
& \quad \leq C\left(\varepsilon \int_{K_{4}}\left|\nabla \psi_{k}(v)\right|^{p(x, t)} \xi^{p^{+}} d x+C(\varepsilon) \int_{K_{4}} \xi^{p^{+}} d x\right. \\
& \left.\quad+\int_{K_{4}} \frac{\xi^{p^{+}}}{\left[k(1+\delta)-(k-v)_{+}+R\right]^{p^{-}-1}} d x\right) .
\end{aligned}
$$

Next, by the previous definition of $k$, the integral involving $\xi_{t}$ is majorised by $\frac{C}{\left(2-p^{-}\right)}$. Therefor, by combining all the previous estimates in (5.15) and using $(5.20)$, we get

$$
\frac{\partial}{\partial t} \int_{K_{4}} \phi_{k}(v) \xi^{p^{+}} d x+C \int_{K_{4}}\left|\nabla \psi_{k}(v)\right|^{p(x, t)} \xi^{p^{+}} d x \leq \frac{C}{2-p^{-}} .
$$

Since $\left|\nabla \psi_{k}(v)\right|^{p^{-}} \leq\left|\nabla \psi_{k}(v)\right|^{p(x, t)}+1$, and using Poincarré's inequality, we obtain

$$
\frac{\partial}{\partial t} \int_{K_{4}} \phi_{k}(v) \xi^{p^{+}} d x+C \int_{K_{4}}\left|\psi_{k}(v)\right|^{p^{-}} \xi^{p^{+}} d x \leq \frac{C}{2-p^{-}} .
$$

Introduce the quantities

$$
Y_{n}=\sup _{-4^{p^{+}} \leq t \leq 0} \int_{K_{4} \cap\left[v(., t)<\delta^{n}\right]} \xi^{p^{+}}(x, t) d x, \quad n=0,1,2, \ldots
$$

Then by using (5.25), Lemma 6.2 in [3] (see also Proposition 7.1 of chapter IV in [10]) gives that for any $\nu \in(0,1)$ being fixed, there exists a constant $\delta \in\left(0, \frac{1}{8}\right)$ depending only on $N, p^{+}, p^{-}, \lambda, \nu$ and such that for all integer $n=1,2, \ldots, i^{*}$ such that

$$
i^{*} \leq \ln R^{-1} \ln ^{-1} \delta^{-1}-\frac{p^{-}}{p^{-}-1}
$$

we have either

$$
Y_{n} \leq \nu
$$

or

$$
Y_{n+1} \leq \max \left\{\nu,(1-\delta) Y_{n}\right\}
$$

Consequently, iterating (5.27)-(5.28) gives Iterating relations (5.25) and (5.26) under the condition (5.24) and using the inequality

$$
Y_{1} \leq\left|K_{4}\right|=2^{N}\left|K_{2}\right|
$$


we obtain

$$
Y_{n} \leq \max \left\{\nu,(1-\delta)^{n-1} Y_{1}\right\} \leq \max \left\{\nu, 2^{N}(1-\delta)^{i-1}\left|K_{2}\right|\right\}, i=2, . ., i^{*}
$$

In (5.31), we choose the smallest $n_{0}$ for which $(1-\delta)^{n_{0}-1} \leq 2^{-N} \nu$ and set $\delta^{n_{0}}=\delta^{*}$. Hence for all $t \in\left[-2^{p^{+}}, 0\right]$, we come to the relation (5.13).

Now we are going to prove Proposition 5.1.

Proof. In Lemma 5.2 choose $\nu=\nu_{0}$ where $\nu_{0}$ is the number claimed by Lemma 4.1, and determine $\delta^{*}=\delta^{*}\left(\nu_{0}\right)$. Let $m_{2}$ defined by

$$
2^{-m_{2}}=\delta^{*}\left(\nu_{0}\right),
$$

and apply Lemma 4.1 with $\mu^{-}=0, \omega=1, m_{1}=0$, and $R=2$ over the boxes

$$
(0, \bar{t})+K_{2} \times\left(-2^{m_{2}\left(p^{-}-2\right)} 2^{p^{+}}, 0\right)=(0, \bar{t})+Q_{2}\left(0, m_{2}\right)
$$

as long as they are contained in $Q_{2}$, i.e., for $\bar{t}$ satisfying

$$
2^{m_{2}\left(p^{-}-2\right)} 2^{p^{+}}-2^{p^{+}} \leq \bar{t} \leq 0 .
$$

Since (5.13) holds true for all time levels in $t \in\left[-2^{+}, 0\right]$, each such box satisfies

$$
\left|\left\{(x, t) \in(0, \bar{t})+Q_{2}\left(0, m_{2}\right) \mid v(x, t) \leq 2^{-m_{2}}\right\}\right| \leq \nu_{0}\left|Q_{2}\left(0, m_{2}\right)\right| .
$$

Therefore, by Lemma 4.1

$$
v \geq 2^{-\left(m_{2}+1\right)}, \forall(x, t) \in(0, \bar{t})+Q_{2}\left(0, m_{2}\right),
$$

for all $\bar{t} \in\left(2^{m_{2}\left(p^{-}-2\right)} 2^{p^{+}}-2^{m_{2}\left(p^{-}-2\right)}, 0\right)$. Since

$$
\left(2^{m_{2}\left(p^{-}-2\right)} 2^{p^{+}}-2^{p^{+}}-2^{m_{2}\left(p^{-}-2\right)}, 0\right) \supset(-1,0) .
$$

We conclude that

$$
v(x, t) \geq 2^{-\left(m_{2}+1\right)}, \quad \forall(x, t) \in Q_{1} .
$$

Consequently, we obtain

$$
u \geq \mu^{-}+\frac{\omega}{2^{m_{2}+2}}, \forall(x, t) \in(\bar{x}, 0)+Q\left(\left(\frac{R}{8}\right)^{p^{+}}, 2 c_{0} R\right),
$$

and the Proposition 5.1 follows with $s_{1}=m_{2}+1$, and $m_{2}=-\log _{2}\left(\delta^{*}\left(\nu_{0}\right)\right)$. 
As an immediate consequence we get the reduction of the oscillation of $u$.

Corollary 5.3. There exists a constant $\sigma_{0} \in(0,1)$ depending only on the data and $\lambda$, such that if (2.9) holds then

$$
\underset{Q\left(\rho^{p^{+}}, c_{0} \rho\right)}{\operatorname{ess} o s c} u \leq \sigma_{0} \omega, \forall \rho \in\left(0, \frac{R}{8}\right) .
$$

Proof. The proof follows since $Q\left(\rho^{p^{+}}, c_{0} \rho\right) \subset Q\left(\left(\frac{R}{8}\right)^{p^{+}}, c_{0} R\right)$, where we take $\sigma_{0}=1-\frac{1}{2^{s_{1}+1}}$.

Assume now that $(2.10)$ holds for all cylinders $(\bar{x}, 0)+Q\left(R^{p^{+}}, d_{0} R\right)$ making up the partition of $Q\left(R^{p^{+}}, c_{0} R\right)$. Since

$$
\mu^{+}-\frac{\omega}{2}=\mu^{-}+\frac{\omega}{2}
$$

we can rephrase $(2.10)$ as

$$
\begin{aligned}
\mid\{(x, t) \in(\bar{x}, 0)+ & \left.Q\left(R^{p^{+}}, d_{0} R\right): u(x, t)>\mu^{+}-\frac{\omega}{2}\right\} \mid \\
\leq & \left(1-\nu_{0}\right)\left|Q\left(R^{p^{+}}, d_{0} R\right)\right|
\end{aligned}
$$

Let $n$ be a positive number to be chosen and arrange that $2^{n \frac{2-p^{-}}{p^{+}}}$is an integer. Then we combine $2^{n N \frac{2-p^{-}}{p^{+}}}$of these cylinders to form boxes congruent to

$$
Q\left(R^{p^{+}}, d_{*} R\right)=K_{d_{*} R} \times\left(-R^{p^{+}}, 0\right), \quad d_{*}=\left(\frac{\omega}{2^{n+1}}\right)^{\frac{p^{-}-2}{p^{+}}}=d_{0} 2^{n \frac{2-p^{-}}{p^{+}}} .
$$

Next, we consider cylinders of type $(\bar{x}, 0)+Q\left(R^{p^{+}}, d_{*} R\right)$. These are contained in $Q\left(R^{p^{+}}, c_{0} R\right)$ if the abscissa $\bar{x}$ of their vertices ranges over the cube $K_{R^{\prime}(\omega)}$, where

$$
\begin{aligned}
R^{\prime}(\omega) & =\left\{2^{\lambda\left(2-p^{-}\right)}-2^{(n+1) \frac{2-p^{-}}{p^{+}}}\right\} \omega^{\frac{p^{-}-2}{p^{+}}} R \\
& =L_{1} d_{*} R
\end{aligned}
$$

where $L_{1}=\left(2^{\lambda-(n+1)}\right)^{\frac{2-p^{-}}{p^{+}}}-1$. We will take $\lambda>n+1$ and arrange that $L_{1}$ is an integer. Then, we regard $Q\left(R^{p^{+}}, c_{0} R\right)$ as the union, up to a set of measure 
zero of $L_{1}^{N}$ pairwise disjoint boxes each congruent to $Q\left(R^{p^{+}}, d_{*} R\right)$. Since each box $(\bar{x}, 0)+Q\left(R^{p^{+}}, d_{*} R\right)$ is the pairwise disjoint union of boxes $(\bar{x}, 0)+$ $Q\left(R^{p^{+}}, d_{0} R\right)$, each of them satisfying (5.38). Therefore, we can rephrase (2.10) this time as

$$
\begin{aligned}
\mid\{(x, t) \in(\bar{x}, 0) & \left.+Q\left(R^{p^{+}}, d_{*} R\right): u(x, t)>\mu^{+}-\frac{\omega}{2}\right\} \mid \\
\leq & \left(1-\nu_{0}\right)\left|Q\left(R^{p^{+}}, d_{*} R\right)\right|
\end{aligned}
$$

for all cylinders $(\bar{x}, 0)+Q\left(R^{p^{+}}, d_{*} R\right)$ making up the partition of $Q\left(R^{p^{+}}, c_{0} R\right)$.

Lemma 5.4. Let $(\bar{x}, 0)+Q\left(R^{p^{+}}, d_{*} R\right)$ be any box contained in $Q\left(R^{p^{+}}, c_{0} R\right)$ and satisfying (5.40). Then, there exists a time level

$$
t^{*} \in\left(-R^{p^{+}},-\frac{\nu_{0}}{2} R^{p^{+}}\right)
$$

such that, for all $s \geq 2$,

$$
\left|\left\{x \in \bar{x}+K_{d_{*} R}, u\left(x, t^{*}\right)>\mu^{+}-\frac{\omega}{2^{s}}\right\}\right| \leq\left(\frac{1-\nu_{0}}{1-\frac{\nu_{0}}{2}}\right)\left|K_{d_{*} R}\right| .
$$

Proof. In fact, if (5.41) doesn't hold, then (5.40) also doesn't hold.

The next lemma asserts that a property similar to (5.41) still holds for all time levels from $t^{*}$ up to 0 .

Lemma 5.5. There exists a positive integer $n$ such that for all $t^{*}<t<0$

$$
\left|\left\{x \in \bar{x}+K_{d_{*} R}: \quad u(x, t)>\mu^{+}-\frac{\omega}{2^{n+1}}\right\}\right| \leq\left(1-\left(\frac{\nu_{0}}{2}\right)^{2}\right)\left|K_{d_{*} R}\right| .
$$

Proof. By translation we may assume that $\bar{x}=0$. Consider the cylinders $K_{d_{*} R} \times\left(t^{*}, 0\right)$ and the level $k=\mu^{+}-\frac{\omega}{2}$. Define

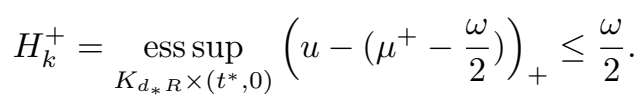

Choose $n \in N$ big enough so that

$$
0<c=\frac{\omega}{2^{n+1}}<H_{k}^{+} .
$$


Then the logarithmic function $\psi^{+}$is well defined and satisfies the following

$$
\begin{gathered}
\psi^{+} \leq n \ln 2, \text { since } \frac{H_{k}^{+}}{H_{k}^{+}-u+k+c} \leq \frac{\frac{\omega}{2}}{c}=2^{n}, \\
0 \leq\left(\psi^{+}\right)^{\prime}=\frac{1}{H_{k}^{+}-u+k+c} \leq \frac{1}{c},
\end{gathered}
$$

and

$$
\left|\left(\psi^{+}\right)^{\prime}\right|^{2-p^{-}} \leq\left(\frac{\omega}{2^{n+1}}\right)^{p^{-}-2}
$$

In the logarithmic inequality (3.11) applied to the function $(u-k)_{+}$let $0<$ $\xi(x) \leq 1$ be a smooth cutoff function defined in $K_{d_{*} R}$ such that for some $\pi \in(0,1)$

$$
\xi=1, \text { on the cube } K_{(1-\pi) d_{*} R} \text {, and }|\nabla \xi| \leq\left(\pi d_{*} R\right)^{-1} .
$$

With these choices, for all $t^{*}<t<0$, we obtain

$$
\begin{aligned}
& \int_{K_{(1-\pi) d_{*} \times\{t\}}}\left[\psi^{+}(u)\right]^{2} d x \leq \int_{K_{d_{*} R} \times\left\{t^{*}\right\}}\left[\psi^{+}(u)\right]^{2} d x \\
& +C\left(\int_{t^{*}}^{0} \int_{K_{d_{*} R}} \psi^{+}(u)\left[\left(\psi^{+}\right)^{\prime}(u)\right]^{2} d x d t\right. \\
& +\left(\frac{1}{\pi d_{*} R}\right)^{p^{+}} \int_{t^{*}}^{0} \int_{K_{d_{*} R}} \psi^{+}(u)\left[\left(\psi^{+}\right)^{\prime}(u)\right]^{2-p^{-}} d x d t \\
& +\left(\frac{1}{\pi d_{*} R}\right)^{p^{+}} \int_{t^{*}}^{0} \int_{K_{d_{*} R}} \psi^{+}(u) d x d t \\
& \left.+\int_{t^{*}}^{0} \int_{K_{d_{*} R}}|u|^{p^{+}} \psi^{+}(u)\left[\left(\psi^{+}\right)^{\prime}(u)\right]^{2-p^{-}} d x d t\right) \\
& \leq \int_{K_{d_{*} R} \times\left\{t^{*}\right\}}\left[\psi^{+}(u)\right]^{2} d x \\
& +C\left(n \ln 2\left(\frac{\omega}{2^{n+1}}\right)^{-2} R^{p^{+}}+n \ln 2\left(\frac{1}{\pi R}\right)^{p^{+}} R^{p^{+}}\right. \\
& \left.+n \ln 2\left(\frac{1}{\pi R}\right)^{p^{+}}\left(\frac{\omega}{2^{n+1}}\right)^{2-p^{-}} R^{p^{+}}+n \ln 2\left(\frac{\omega}{2^{n+1}}\right)^{-2} R^{p^{+}}\right)\left|K_{d_{*} R}\right| \\
& \leq n^{2}(\ln 2)^{2}\left(\frac{1-\nu_{0}}{1-\frac{\nu_{0}}{2}}\right)\left|K_{d_{*} R}\right|+\frac{C n}{\pi^{p^{+}}}\left|K_{d_{*} R}\right|,
\end{aligned}
$$

where we used Lemma 5.4 and the assumptions (2.6) and (4.2). 
The left hand side is estimated below by integrating over the smaller set

$$
\left\{x \in K_{(1-\pi) d_{*} R}: u(x, t)>\mu^{+}-\frac{\omega}{2^{n+1}}\right\} .
$$

On such a set, $\xi=1$ and since $\psi$ is a decreasing function of $H_{k}^{+}$, we have

$$
\psi^{2} \geq \ln ^{2}\left(\frac{\frac{\omega}{2}}{\frac{\omega}{2^{1+n-1}}}\right)=(n-1)^{2} \ln ^{2} 2 .
$$

We carry this in the previous estimation and divide by $(n-1)^{2} \ln ^{2} 2$, to obtain for all $t^{*}<t<0$

$$
\begin{aligned}
\mid\left\{x \in K_{(1-\pi) d_{*} R}:\right. & \left.u(x, t)>\mu^{+}-\frac{\omega}{2^{n+1}}\right\} \mid \\
\leq & {\left[\left(\frac{n}{n-1}\right)^{2}\left(\frac{1-\nu_{0}}{1-\frac{\nu_{0}}{2}}\right)+\frac{C}{n \pi^{p^{+}}}\right]\left|K_{d_{*} R} \cdot\right| }
\end{aligned}
$$

On the other hand

$$
\begin{aligned}
& \left|\left\{x \in K_{d_{*} R}, u(x, t)>\mu^{+}-\frac{\omega}{2^{n+1}}\right\}\right| \\
& \leq\left|\left\{x \in K_{(1-\pi) d_{*} R}: u(x, t)>\mu^{+}-\frac{\omega}{2^{n+1}}\right\}\right|+N \pi\left|K_{d_{*} R}\right| \\
& \leq\left\{\left(\frac{n}{n-1}\right)^{2}\left(\frac{1-\nu_{0}}{1-\frac{\nu_{0}}{2}}\right)+\frac{C}{n \pi^{p^{+}}}+N \pi\right\}\left|K_{d_{*} R}\right| .
\end{aligned}
$$

The proof is complete once we choose $\pi$ so small that $N \pi \leq \frac{3}{8} \nu_{0}^{2}$, then $\mathrm{n}$ so large that

$$
\frac{C}{n \pi^{p^{+}}} \leq \frac{3}{8} \nu_{0}^{2} \text { and }\left(\frac{n}{n-1}\right)^{2} \leq\left(1-\frac{\nu_{0}}{2}\right)\left(1+\nu_{0}\right)>1
$$

The information of Lemma 5.5 will be exploited to show that in a small cylinder about $(0,0)$, the solution $u$ is strictly bounded above by

$$
\mu^{+}-\frac{\omega}{2^{m}}, \text { for some } m>n+1
$$

The process also determines the number $\lambda$ that defines the size of $Q\left(R^{p^{+}}, c_{0} R\right)$. To make this quantitative consider the box

$$
Q\left(\beta R^{p^{+}}, c_{0} R\right), \beta=\frac{\nu_{0}}{2}, c_{0}=\left(\frac{\omega}{2^{\lambda}}\right)^{\frac{p^{-}-2}{p^{+}}} .
$$


We view $Q\left(\beta R^{p^{+}}, c_{0} R\right)$ as partitioned into sub-boxes $(\bar{x}, 0)+Q\left(\beta R^{p^{+}}, d_{*} R\right)$ where $\bar{x}$ takes finitely many points within the cube $K_{R^{\prime}(\omega)}$. For each of these sub-cylinders Lemma 5.5 holds.

Lemma 5.6. For every $\nu \in(0,1)$ there exists a number $m$ depending only on the data and independent of $\omega$ and $R$ such that, for all cylinders $(\bar{x}, 0)+$ $Q\left(\beta R^{p^{+}}, d_{*} R\right)$ making up the partition of $Q\left(\beta R^{p^{+}}, c_{0} R\right)$, we have

$$
\begin{aligned}
\mid\{(x, t) \in(\bar{x}, 0) & \left.+Q\left(\beta R^{p^{+}}, d_{*} R\right): u(x, t)>\mu^{+}-\frac{\omega}{2^{m}}\right\} \mid \\
& \leq \nu\left|Q\left(\beta R^{p^{+}}, d_{*} R\right)\right|
\end{aligned}
$$

Proof. After a translation we may assume that $(\bar{x}, 0)=(0,0)$. Set $s_{0}=n+1$ and consider the energy inequality $(3.1)$ writhen for $(u-k)_{+}$,

$$
k=\mu^{+}-\frac{\omega}{2^{s}}, s=s_{0}, s_{0}+1, s_{0}+2, . ., m-1
$$

over the cylinder $Q\left(\beta(2 R)^{p^{+}}, 2 d_{*} R\right)$. Over such a box

$$
\left(u(x, t)-\left(\mu^{+}-\frac{\omega}{2^{s}}\right)\right)_{+} \leq \frac{\omega}{2^{s}} \text { a.e. }(x, t) \in Q\left(\beta(2 R)^{p^{+}}, 2 d_{*} R\right) .
$$

The cutoff function $(x, t) \longrightarrow \xi(x, t)$ is taken to satisfy

$$
\left\{\begin{array}{l}
\xi=1, \text { on } Q\left(\beta R^{p^{+}}, d_{*} R\right) \\
\xi=0, \text { on the parabolic boundary of } Q\left(\beta(2 R)^{p^{+}}, 2 d_{*} R\right), \\
|\nabla \xi| \leq\left(\frac{1}{d_{*} R}\right)^{\frac{p^{-}}{p^{+}}}, 0 \leq \xi_{t} \leq \frac{2}{\nu_{0} R^{p^{-}}}
\end{array}\right.
$$

We put these estimates in (3.1) and discard the first non-negative term on the 
left hand side. This gives

$$
\begin{aligned}
&\left.\iint_{Q\left(\beta R^{\left.p^{+}, d_{*} R\right)} \mid\right.} \nabla(u-k)_{+}\right|^{p^{-}} d x d t \\
& \leq C\left[\frac{2}{\nu_{0} R^{p^{-}}} \iint_{Q\left(\beta(2 R)^{\left.p^{+}, 2 d_{*} R\right)}\right.}(u-k)_{+}^{2} d x d t\right. \\
&+ \frac{1}{\left(d_{*} R\right)^{p^{-}}} \iint_{Q\left(\beta(2 R)^{\left.p^{+}, 2 d_{*} R\right)}\right.}(u-k)_{+}^{p^{+}} d x d t \\
&+\left.\iint_{Q\left(\beta(2 R)^{p^{+}}, 2 d_{*} R\right)} \chi\left((u-k)_{+}>0\right) d x d t\right] \\
& \leq C\left[\frac{2}{R^{p^{-}}}\left(\frac{\omega}{2^{s}}\right)^{2}+\left(\frac{1}{d_{*} R}\right)^{p^{-}}\left(\frac{\omega}{2^{s}}\right)^{p^{+}}\right. \\
&+\left.2^{n+p^{+}}\left(\frac{1}{d_{*} R}\right)^{p^{-}}\left(\frac{\omega}{2^{s}}\right)^{p^{-}}\left(\frac{1}{d_{*} R}\right)^{-p^{-}}\left(\frac{\omega}{2^{s}}\right)^{-p^{+}}\right] \\
& \times\left|Q\left(\beta R^{p^{+}}, d_{*} R\right)\right| \cdot
\end{aligned}
$$

Since $\omega \leq 1$, then

$$
\begin{aligned}
\left(\frac{\omega}{2^{s}}\right)^{2} & =\left(\frac{\omega}{2^{s}}\right)^{2-p^{-}}\left(\frac{\omega}{2^{s}}\right)^{p^{-}} \leq\left(\frac{\omega}{2^{n+1}}\right)^{2-p^{-}}\left(\frac{\omega}{2^{s}}\right)^{p^{-}} \\
& =\left(\frac{\omega}{2^{n+1}}\right)^{\left(\frac{2-p^{-}}{p^{+}}\right) p^{+}}\left(\frac{\omega}{2^{s}}\right)^{p^{-}} \leq\left(\frac{1}{d_{*}}\right)^{p^{-}}\left(\frac{\omega}{2^{s}}\right)^{p^{-}},
\end{aligned}
$$

and by means of the assumption (2.6) and Remark 2.5 we may estimate

$$
\begin{aligned}
\left(\frac{\omega}{2^{s}}\right)^{-p^{-}} R^{p^{-}} d_{*}^{p^{-}} & \leq\left(\frac{\omega}{2^{s}}\right)^{-p^{-}+\frac{\left(p^{-}-2\right) p^{-}}{p^{+}}} R^{p^{-}} \\
& \leq 1
\end{aligned}
$$

Therefore, we obtain

$$
\begin{aligned}
\iint_{Q\left(\beta R^{\left.p^{+}, d_{*} R\right)}\right.}\left|\nabla(u-k)_{+}\right|^{p^{-}} d x d t \\
\leq \frac{C}{\left(d_{*} R\right)^{p^{-}}}\left(\frac{\omega}{2^{s}}\right)^{p^{-}}\left|Q\left(\beta R^{p^{+}}, d_{*} R\right)\right| .
\end{aligned}
$$

Now, we consider the levels

$$
l=\mu^{+}-\frac{\omega}{2^{s+1}}, k=\mu^{+}-\frac{\omega}{2^{s}}, s \geq s_{0} .
$$


By virtue of Lemma 5.5, we get

$$
\left|\left\{x \in K_{d_{*} R}, u(., t)<\mu^{+}-\frac{\omega}{2^{s}}\right\}\right| \geq\left(\frac{\nu_{0}}{2}\right)^{2}\left|K_{d_{*} R}\right|, \forall t \in\left(-\beta R^{p^{+}}, 0\right) .
$$

Setting

$$
A_{s}(t)=\left\{x \in K_{d_{*} R}, u(., t)>\mu^{+}-\frac{\omega}{2^{s}}\right\}, A_{s}=\int_{-\beta R^{p^{+}}}^{0}\left|A_{s}(t)\right| d t .
$$

Then, from Lemma 2.2 of chap I in [10], we obtain

$$
\left(\frac{\omega}{2^{s+1}}\right)\left|A_{s+1}(t)\right| \leq \frac{C}{\nu_{0}^{2}} d_{*} R \int_{A_{s}(t) \backslash A_{s+1}(t)}|\nabla u| d x, \quad \forall t \in\left(-\beta R^{p^{+}}, 0\right) .
$$

Let us integrate both sides of (5.49) over the interval $\left(-\beta R^{p^{+}}, 0\right)$. Then, by using Hölder's inequality and raising the resulting relation to the power $p^{-}$, we obtain

$$
\begin{aligned}
& \left(\frac{\omega}{2^{s+1}}\right)^{p^{-}}\left|A_{s+1}(t)\right|^{p^{-}} \\
& \quad \leq C\left(d_{*} R\right)^{p^{-}}\left(\iint_{A_{s}}|\nabla u|^{p^{-}} d x\right)\left|A_{s} \backslash A_{s+1}\right|^{p^{-}-1} \\
& \quad \leq C\left(d_{*} R\right)^{p^{-}} \frac{1}{\left(d_{*} R\right)^{p^{-}}}\left(\frac{\omega}{2^{s}}\right)^{p^{-}}\left|Q\left(\beta R^{p^{+}}, d_{*} R\right)\right|\left|A_{s} \backslash A_{s+1}\right|^{p^{-}-1} .
\end{aligned}
$$

Then,

$$
\left|A_{s+1}\right|^{\frac{p^{-}}{p^{-}-1}} \leq C\left|Q\left(\beta R^{p^{+}}, d_{*} R\right)\right|^{\frac{1}{p^{-}-1}}\left|A_{s} \backslash A_{s+1}\right|,
$$

for all $s=s_{0}, s_{0}+1, \ldots, m-1$. Since $\mu^{+}-\frac{\omega}{2^{s+1}} \leq \mu^{+}-\frac{\omega}{2^{m}}$, the quantities $\left|A_{s+1}\right| \geq\left|A_{m}\right|$. We combine this fact to obtain

$$
\sum_{s=s_{0}}^{m-1}\left|A_{s+1}\right|^{\frac{p^{-}}{p^{-}-1}} \geq\left(m-s_{0}\right)\left|A_{m}\right|^{\frac{p^{-}}{p^{-}-1}} .
$$

Note also that $\sum_{s=s_{0}}^{m-1}\left|A_{s} \backslash A_{s+1}\right| \leq\left|Q\left(\beta R^{p^{+}}, d_{*} R\right)\right|$. Collecting these estimates, we arrive at

$$
\left|A_{m}\right| \leq \frac{C}{\left(m-s_{0}\right)^{\frac{p^{-}-1}{p^{-}}}}\left|Q\left(\beta R^{p^{+}}, d_{*} R\right)\right|,
$$


and the proof is complete once we choose $m$ sufficiently large so that

$$
\left(\frac{C}{m-s_{0}}\right)^{\frac{p^{-}-1}{p^{-}}} \leq \nu .
$$

To proceed we return to the box $Q\left(\beta R^{p^{+}}, c_{0} R\right)$ and recall that it is the finite union, up to a set of measure zero, of pairwise disjoint boxes $(\bar{x}, 0)+$ $Q\left(\beta R^{p^{+}}, d_{*} R\right)$. Therefore Lemma 5.6 implies.

Corollary 5.7. For every $\nu \in(0,1)$, there exists a number $m$ depending only upon the data and independent of $\omega$ and $R$ such that

$$
\begin{gathered}
\left|\left\{(x, t) \in Q\left(\beta R^{p^{+}}, c_{0} R\right): u(x, t)>\mu^{+}-\frac{\omega}{2^{m}}\right\}\right| \\
\leq \nu\left|Q\left(\beta R^{p^{+}}, c_{0} R\right)\right| .
\end{gathered}
$$

We finally determine the size of the cylinder $Q\left(\beta R^{p^{+}}, c_{0} R\right)$ and consequently the number $\lambda$. First, in Corollary 5.7 take $\nu=\nu_{0}$ and determine $m$ accordingly. Then let $m_{2}$ be given by

$$
\beta=\frac{\nu_{0}}{2}=2^{m_{2}\left(p^{-}-2\right)},
$$

and assume that $m \geq m_{2}$. Determine $\lambda$ from

$$
\lambda=m_{1}, \text { and } m=m_{1}+m_{2} \text {. }
$$

With these choices, the cylinder $Q\left(\beta R^{p^{+}}, c_{0} R\right)$ coincides with the cylinder $Q\left(m_{1}, m_{2}\right)$ introduced in Lemma 4.1. By Corollary 5.7, we have

$$
\left|\left\{(x, t) \in Q_{R}\left(m_{1}, m_{2}\right): u(x, t)>\mu^{+}-\frac{\omega}{2^{m}}\right\}\right| \leq \nu_{0}\left|Q_{R}\left(m_{1}, m_{2}\right)\right|,
$$

Using Lemma 4.1 implies

$$
u(x, t) \leq \mu^{+}-\frac{\omega}{2^{m+1}}, \quad \forall(x, t) \in Q_{\frac{R}{2}}\left(m_{1}, m_{2}\right) .
$$

which leads us to the following statement.

Proposition 5.8. Assume that (2.10) holds true for all cylinders $(\bar{x}, 0)+$ $Q\left(R^{p^{+}}, d_{0} R\right)$ making up the partition of $Q\left(R^{p^{+}}, c_{0} R\right)$. Then, for all $0<$ $\rho<\frac{R}{2}$

$$
\underset{Q\left(\beta \rho^{p^{+}}, c_{0} \rho\right)}{\operatorname{esc}} u \leq \sigma_{1} \omega,
$$

where $\sigma_{1}=1-\frac{1}{2^{m+1}}$. 
Now, we are going to prove our main result Proposition 2.6.

Proof. The proof of Proposition 2.6 follows by combining the two alternatives. The conclusion of the first alternative is that

$$
\underset{Q\left(\rho^{p^{+}}, c_{0} \rho\right)}{\operatorname{ess} \operatorname{osc}} u \leq \sigma_{0} \omega, \quad \forall \rho \in\left(0, \frac{R}{8}\right),
$$

where $\sigma_{0}=1-\frac{1}{2^{s_{1}+1}}$. The conclusion of the second alternative is that

$$
\underset{Q\left(\beta \rho^{p^{+}}, c_{0} \rho\right)}{\operatorname{ess} \operatorname{osc}} u \leq \sigma_{1} \omega, \quad \forall \rho \in\left(0, \frac{R}{2}\right),
$$

where $\sigma_{1}=1-\frac{1}{2^{m+1}}$. Set

$$
\sigma=\max \left\{\sigma_{0}, \sigma_{1}\right\}, \text { and } C=\frac{\beta^{\frac{1}{p^{+}}}}{4}=\left(\frac{\nu_{0}}{2^{2 p^{+}+1}}\right)^{\frac{1}{p^{+}}}
$$

Observe that, assuming $\nu_{0} \leq \frac{1}{2}$

$$
c \leq \frac{1}{2^{2+\frac{2}{p^{+}}}}<\frac{1}{8}<\frac{1}{2}, \text { and } C \leq \frac{\beta^{\frac{1}{p^{+}}}}{2} .
$$

Define

$$
R_{1}=C R, \quad \omega_{1}=\sigma \omega
$$

and the cylinder

$$
Q_{1}=Q\left(R_{1}^{p^{+}}, C_{1} R_{1}\right), \quad C_{1}=\left(\frac{\omega_{1}}{2^{\lambda}}\right)^{\frac{p^{-}-2}{p^{+}}}
$$

Since $\sigma<1$,

$$
C_{1} R_{1} \leq c_{0} R_{1}
$$

Therefore, combining both alternatives,

$$
\underset{Q_{1}}{\operatorname{ess} \text { osc }} u \leq \omega_{1}
$$

The process can now be repeated inductively starting from such relation. 


\section{References}

[1] E. Acerbi, G. Mingione and G.A. Seregin, Regularity results for parabolic systems related to a class of non-Newtonian fluids, Ann. Inst. H. Poincaré Anal. Non Linéaire, 21 (2004), 25-60.

[2] E. Acerbi, G. Mingione, Regularity results for stationary electrorheological fluids, Arch. Ration. Mech. Anal. 164 (2002) 213-259.

[3] Yu. A. Alkhutov, V. V. Zhikov; Hölder continuity of solutions of parabolic equations with variable nonlinearity exponent, Translation of Tr. Semin. im. I. G. Petrovskogo, No.28 (2011), Part I, 8-74, J. Math. Sci. (N.Y.), V. 179, (2011), 347-389.

[4] S. Antontsev and S. Shmarev, Evolution PDEs with Nonstansdard Growth Conditions, Atlantis Press, Amsterdam, 2015.

[5] S. Antontsev, J. I. Díaz, and S. Shmarev, Energy methods for free boundary problems: Applications to nonlinear PDEs and fluid mechanics, Progress in Nonlinear Differential Equations and their Applications, vol. 48 (2002) Birkhäuser, Boston.

[6] S. Antontsev and S. Shmarev, Parabolic equations with anisotropic nonstandard growth conditions, Internat. Ser. Numer. Math., vol. 154 (2007) Birkhäuser, Basel, 33-44 .

[7] H. El Bahja, Hölder regularity for degenerate parabolic equations with variable exponents, Bol. Soc. Paran. Mat, In press, Available online August 14, (2018).

[8] H. El Bahja, Regularity for anisotropic quasi-linear parabolic equations with variable growth, Electron.J.Differential Equations, Vol.2019 (2019), No. 104, pp.1-21.

[9] Y. Chen, S. Levine and M. Rao, Variable exponent, linear growth functionals in image restoration, SIAM J. Appl. Math. 66 (2006), 1383-1406.

[10] E. DiBenedetto, Degenerate Parabolic Equations. Springer-Verlag, New York, 1993.

[11] E. DiBenedetto, J.M. Urbano, V. Vespri, Current issues on singular and degenerate evolution equations. in: Handbook of Differential Equations, Evolutionary Equations, vol. 1, (2004), pp. 169-286. 
[12] E. De Giorgi; Sulla differenziabilità e l'analicità delle estremali degli integrali multipli regolari, Mem. Accad. Sci. Torino Cl. Sci. Fis. Mat. Natur, 3 (3)(1957) 25-43.

[13] M. Marin, O. Florea, On temporal behavior of solutions in Thermoelasticity of porous micropolar bodies, An. Sti. Univ. Ovidius Constanta, Vol.22, issue 1,(2014), 169-188

[14] J. Moser, A new proof of De Giorgi's theorem concerning the regularity problem for elliptic differential equations, Comm. Pure Appl. Math,13 (1960) 457-468.

[15] J. Moser, On Harnack's theorem for elliptic differential equations,Comm. Pure Appl. Math,14(1961) 577-591.

[16] J. Moser, A Harnack inequality for parabolic differential equations,Comm. Pure Appl. Math, 17 (1964) 101-134.

[17] J. Moser, Correction to A Harnack inequality for parabolic differential equations, Comm. Pure Appl. Math, 20(1967) 231-236.

[18] J. Nash, Continuity of solutions of parabolic and elliptic equations,Amer. J. Math, 80(1958) 931-954.

[19] S. Ouaro, A. Ouedraogo; Nonlinear parabolic problems with variable exponent and L1-data, Electron. J. Differential Equations, Vol. 2017 (2017), No. 32 , pp. 1-32.

[20] K. Rajagopal, M. Ruzicka, Mathematical modelling of electro-rheological fluids, Contin. Mech. Thermodyn. 13 (2001) 59-78.

[21] M. Ruzicka, Electrorheological Fluids: Modeling and Mathematical Theory, Lecture Notes in Mathematics, vol. 1748, Springer, Berlin, 2000.

[22] J.M, Urbano.: The method of intrinsic scaling. A systematic approach to regularity for degenerate and singular PDEs. Lecture Notes in Mathematics, vol. 1930. Springer, Berlin (2008).

[23] P. Wittbold, A. Zimmermann, Existence and uniqueness of renormalized solutions to non- linear elliptic equations with variable exponents and L1data, Nonlinear Analysis TMA, 72 (2010), 2990-3008.

[24] V.V. Zhikov, On the density of smooth functions in Sobolev-Orlicz spaces, Zap. Nauchn. Sem. S. Peterburg. Otdel. Mat. Inst. Steklov. (POMI) 310 (2004), 67-81. 
[25] C. Zhang, S. Zhou., Renormalized and entropy solutions for nonlinear parabolic equations with variable exponents and L1-data, J. Differ. Equations, 248 (2010), 1376-1400.

Hamid EL BAHJA,

Laboratory of Mathematical Analysis and Applications,

Department of Mathematics, P.O. Box 1014, Faculty of Sciences,

Mohammed V University, Rabat, Morocco.

Email: hamidsm88@gmail.com 